\title{
Elastic and Anelastic Behavior of Microwave Sintered BCT-BST Ceramics
}

\author{
C. Pavithra, W. Madhuri, S. Roopas Kiran, N. Arunai Nambi Raj, K. V. Siva Kumar
}

\begin{abstract}
Lead free 0.55(Ba0.9Ca0.1) TiO3-0.45Ba (Sn0.2Ti0.8) O3 (BCT-BST) ceramic is synthesized by three different techniques viz solid state method, sol-gel method and molten-salt method. The prepared BCT-BST ceramic samples exhibited cubic crystal structure on $X$-ray powder diffraction analysis. The morphology of the samples is analyzed using transmission electron microscope. The lattice interactions with ultrasonic waves and morphotropic phase boundary at $70^{\circ} \mathrm{C}$ is confirmed from elastic and anelastic studies of the ceramic. High piezoelectric coefficient $d 33$ of $623 \mathrm{pC} / \mathrm{N}$ is achieved in the prepared BCT-BST ceramics.
\end{abstract}

Keywords: Sol-gel method, Solid state method, Molten-salt method, internal friction, longitudinal modulus.

\section{INTRODUCTION}

The invention of lead zirconate titanate (PZT) has triggered the industrial revolution and has played a vital role in technological advancement. Very high piezoelectric constant of PZT single crystals and polycrystalline PZT has enhanced the electromechanical transducer efficiency [1]. Single crystal PZT exhibits a d33 of about 2000 to $2500 \mathrm{pC} /$ while the polycrystalline PZT exhibits in the range of 600 to $700 \mathrm{pC} / \mathrm{N}$. Since past few decades, most of the European countries have passed the bill against the usage of lead based materials. This has stimulated the scientist community to develop high d33 lead free piezoelectric ceramics. In the pursuit barium titanate has been extensively studied with various compositional and structural modifications [2]. Systems like NBT, BNT-NT, KNN, KNBT, BST, BCST etc were studied and none could record a d33 greater than PZT. Li Fung et al [3] have studied systematically a series of BCT-xBST compositions. The compositions were prepared by conventional synthesis at $1480^{\circ} \mathrm{C}$. The present composition of $0.55 \mathrm{BCT}-0.45 \mathrm{BST}$ is reported to have a d33 of $630 \mathrm{pC} / \mathrm{N}$ [3]. In order to study the elastic and anelastic nature of ceramic samples, composite oscillator technique is employed. In the technique ultrasonic waves are propagated through the ceramics. Behaviour of ultrasonic waves

Revised Manuscript Received on December 20, 2019

* Correspondence Author

W. Madhuri*, ${ }^{2}$ Ceramic Composites Laboratory, CCG, VIT, Vellore-632014, India. madhuriw12@gmail.com

C. Pavithra, Department of Physics, Marudhar Kesari Jain Collage, Vaniyamadi, Vellore Email: paviselvi91@gmail.com

S. Roopas Kiran, Department of Physics, VIT-AP. Email: roopasiitm@gmail.com

Arunai Nambiraj N , Center for Biomaterilas Cellular \& Molecular Theranostics, VIT, Vellore-632014Email: narunainambiraj@vit.ac.in

K. V. Siva Kumar, Ceramic Composite Materials Laboratory,

Department of Physics, Sri Krishnadevaraya University, Anantapur-515003, AP Email: sivakumar.sivani@gmail.com propagating through solid ceramic sample can reveal the lattice properties of the sample. The acoustic waves propagated directly interact with lattice of the ceramic. The acoustic wave velocity is very sensitive to crystal lattice defects, imperfections and lattice phase boundary transitions of the ceramics [4 - 9]. Manson et al and others have extensively investigated the elastic behavior of solids. [10 14]. Based on the internal friction values the ceramics have applications as sound delay lines. In the present article the synthesis of BCT-BST ceramic by solid state reaction route, molten salt technique and sol gel followed by microwave synthesis is discussed along with their d33 measurements. Sharp morphotropic phase boundary observed from elastic and anelastic behavior of ceramics is reported.

\section{EXPERIMENTAL DETAILS}

\subsection{Solid State Reaction}

Barium carbonate (BaCO3, Sdfine 99.0\%), calcium carbonate $(\mathrm{CaCO} 3$, Alfa Aesar $99 \%)$, stannic oxide $(\mathrm{SnO} 2$, Alfa Aesar 99.5\%) and titanium dioxide (TiO2, Alfa Aesar 99.0\%) are used as raw materials. The starting materials are taken in stoichiometric ratio and grinded in an agate mortar for uniform mixing. The powders are transferred into tungsten carbide jar (the sample and ball ratio is 1:10) and is grinded for $6 \mathrm{~h}$ in ethanol medium, using Fritsch planetary mono mill. The finely grinded green powder is calcined at $1300{ }^{\circ} \mathrm{C}$ for $4 \mathrm{~h}$ using conventional furnace. The resultant powder is compacted into a parallelepiped bar of dimensions $20 \mathrm{~mm} \times 4 \mathrm{~mm} \times 4 \mathrm{~mm}$ using PVA as a binder and sintered using microwave furnace at $1400{ }^{\circ} \mathrm{C}$ for $30 \mathrm{~min}$. The sintered bar is glued to a quartz transducer of same dimensions to form the composite oscillator.

\subsection{Sol-gel synthesis procedure}

Initially, barium acetate is mixed with glacial acetic acid and stirred continuously. Later the alcoholic solutions of calcium nitrate and stannic chloride (tin chloride) are prepared independently. All the three solutions are mixed together with continuous stirring for $30 \mathrm{~min}$. and titanium butoxide is added to the solution slowly. The solution is dehydrated at $100^{\circ} \mathrm{C}$ by keeping it in a hot air oven. The obtained white gel is dried to obtain the powder. The dry powder is calcined at $1000{ }^{\circ} \mathrm{C}$ while the final sintering temperature of the bar is $1250^{\circ} \mathrm{C}$ using microwave furnace.

\subsection{Molten-salt synthesis}

Essentially in this method barium carbonate $(\mathrm{BaCO} 3$, Sdfine $99.0 \%$ ), calcium carbonate (CaCO3, Alfa Aesar 99\%), stannic oxide ( $\mathrm{SnO} 2$, Alfa Aesar 99.5\%), titanium dioxide (TiO2, Alfa Aesar 99\%), electrolytic mixture sodium chloride $(\mathrm{NaCl}$, Sdfine, $98 \%)$

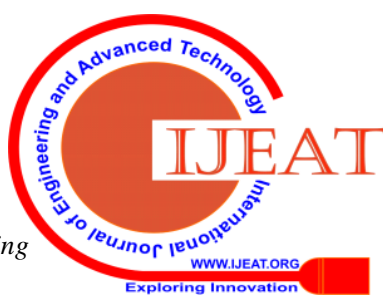


and potassium chloride $(\mathrm{KCl}$, Sdfine, $98 \%)$ are used as raw materials in stoichiometric ratio. Initially, all the salts are mixed using an agate mortar. The ratio of oxide to chloride salts is 1:1. The oxide and chloride salts are grinded in planetary mono mill for 8 hours and is calcined for $1000^{\circ} \mathrm{C}$. The calcined sample is washed for several times using deionized water to remove the salt ions until $\mathrm{Cl}$ - is not detected using AgNO3 solution. The washed powder is heated at $150^{\circ} \mathrm{C}$ using hot air oven to obtain the dry powder. The bar sample prepared is sintered at $1250^{\circ} \mathrm{C}$ using microwave furnace.

\section{CHARACTERIZATION DETAIL}

Crystal structure and crystallite size were analyzed by X-ray powder diffraction (XRPD) method using (D8 Advance, Bruker diffractometer). The morphology of the samples is analyzed by Transmission Electron Microscope (Technai, G2 20 Twin). The piezoelectric coefficient d33 is measured by Sinocera piezotronics (YE2730A). The elastic and inelastic property is measured using the piezoelectric composite oscillator by Mittal Enterprises Pvt. Ltd. The details of the experimental setup is given in Ramana et al [15].

\section{RESULTS AND DISCUSSION}

\subsection{Structural and morphology analysis}

The phase formation of BCT-BST is studied using X-ray powder diffraction technique (XRPD). Fig. 1 shows the XRPD profile peaks of BCT-BST and all peaks are well matched with the reported literature $[3,16]$. The single phase formation of BCT-BST exhibits cubic crystal structure.

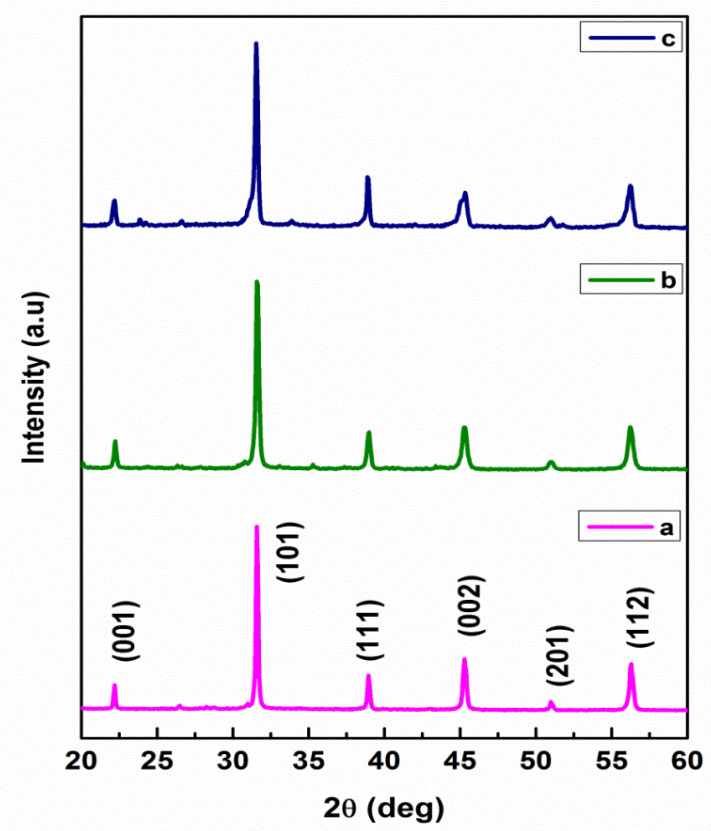

Fig. 1.XRPD pattern of BCT-BST ceramics synthesized by (a) SSR (b) Sol-Gel Method and (c) Molten-Salt Method

The cubic crystal structure exhibited in the present ceramic samples is mainly due to the replacement of Ti4+ with Sn4+ and in good agreement with Zhu et al [3]. The density is calculated using the density meter. On observation it is evident that obtained densities are in good agreement with the theoritical densities and the calculated porosity is only $3 \%$ for all the ceramic samples. The theoritical density is evaluated from XRPD analysis and are tabulated in Table 1. Densities equal to the theoritical density affirms the estimation of the densification temperature for different synthesis techniques. The average crystallite size is calculated using the Scherrer formula. It is noticed that SSR technique yields large crystals compared to the other two methods (values in Table. 1). The Scherrer strain is calculated using the relation (1).

$$
\varepsilon^{*}=\frac{\beta}{4 \tan \theta}
$$

Fig. 2 shows the Williams-Hall (W-H) plot for SSR, sol-gel method and molten-salt technique. From the W-H plot the strain is calculated and when compared to Scherrer strain are found to be within the acceptable error limits (tabulated in Table 1). The crystallite size and lattice strains (as observed from XRD) depend on synthesis and sintering techniques. The BCT-BST has exhibited an increased strain in the synthesis order of SSR, sol-gel and molten salt. This indicates that the crystal lattice suffers less stain in SSR method compared to the other two techniques. The present BCT-BST via SSR has exhibited a larger crystallite size of $(\sim 60 \mathrm{~nm})$ while experienced the lowest strain. A similar behavior is observed for the BCT-BST obtained by sol-gel and molten salt. The crystallite size and strain have exhibited an inverse relation.

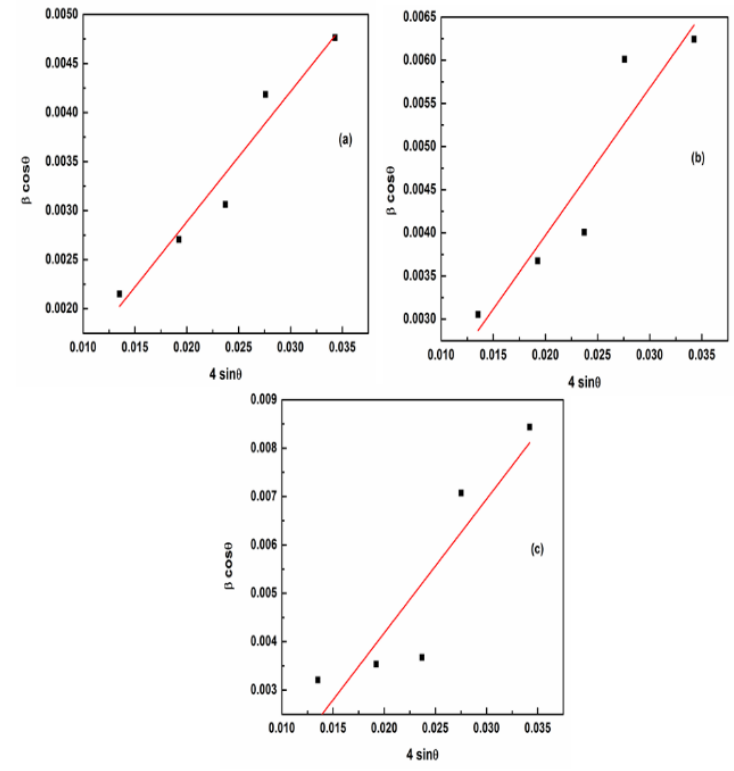

Fig. 2. W-H plot for (a) SSR, (b) Sol-gel method (c) Molten-salt method synthesized BCT-BST ceramics.

Fig. 3 shows the transmission electron microscope (TEM) images of all the prepared samples. It is noticed that the powder is highly agglomerated which must be due to the columbic interactions of nanoparticles. However no particular geometry is noticed from the TEM pictures. Ceramics synthesized by sol gel technique exhibited the smallest particle size of $32 \mathrm{~nm}$ (Table. 1). Fig. 4 shows the selected area electron diffraction (SAED) pattern of BCT-BST. The SAED images of sol-gel and molten-salt samples reveal single crystal

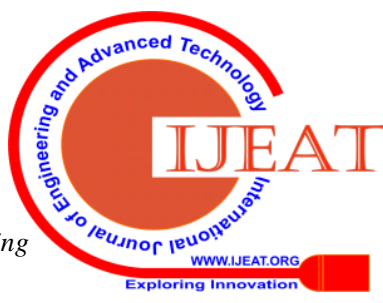


nature [17] of the particles while SSR technique resulted in polycrystalline ceramics. The structural and morphological results discussed till now confirms the high quality of ceramics prepared and best skills of the authors in synthesis techniques adopted.

\subsection{Piezoelectric coefficient $\left(d_{33}\right)$, Piezoelectric Voltage \\ $\left(\mathbf{G}_{33}\right)$}

The prepared samples are poled for $30 \mathrm{~min}$ at room temperature; in an electric field of $1 \mathrm{kV} / \mathrm{cm}$. The poled samples are used to measure the piezoelectric coefficient. The piezoelectric coefficient $\left(\mathrm{d}_{33}\right)$ of prepared samples are noticed to be independent of the density, porosity and lattice stains. For solid state, sol-gel and molten-salt method synthesized BCT-BST $d_{33}$ values are in (Table 1) the range of 619 to $623 \mathrm{pC} / \mathrm{N}$. The analysis, the $\mathrm{d} 33$ seems to be more for large crystalline or grained ceramics compared to smaller crystallite or grained ceramics. The piezoelectric voltage $\mathrm{G}_{33}$ is measured from the below relation (2)

$$
G_{33}=\frac{d_{33}}{\varepsilon_{33}}
$$

where $\mathrm{d} 33$ is the observed piezoelectric coefficient and $\varepsilon_{33}$ is the lattice dielectric constant at room temperature. The calculated values of piezoelectric voltage is tabulated in Table 1.

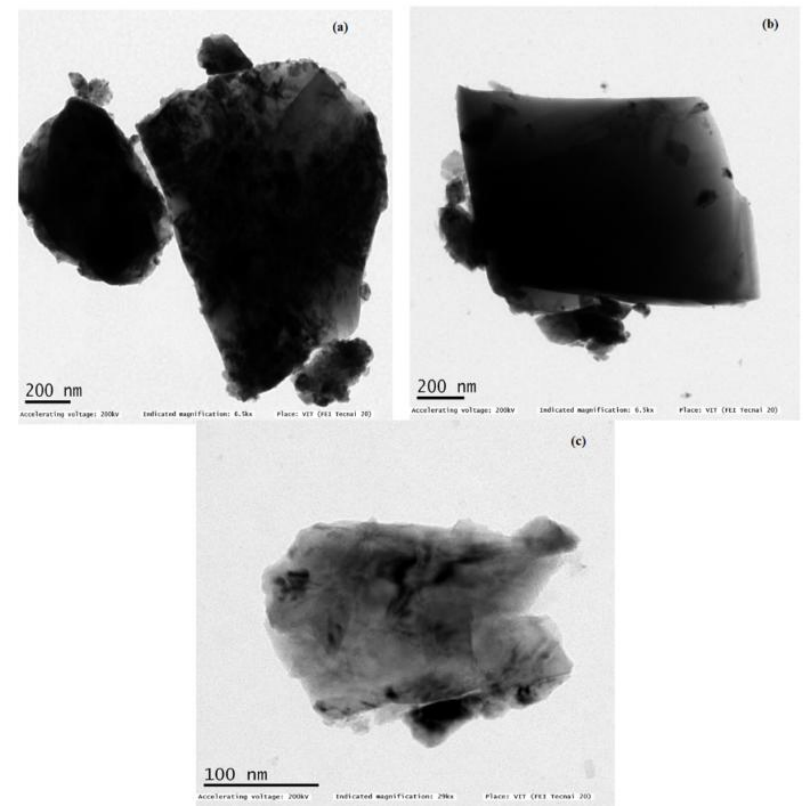

Fig. 3. TEM images of BCT-BST synthesized by (a) SSR, (b) Sol-Gel Method and (c) Molten-Salt Method.

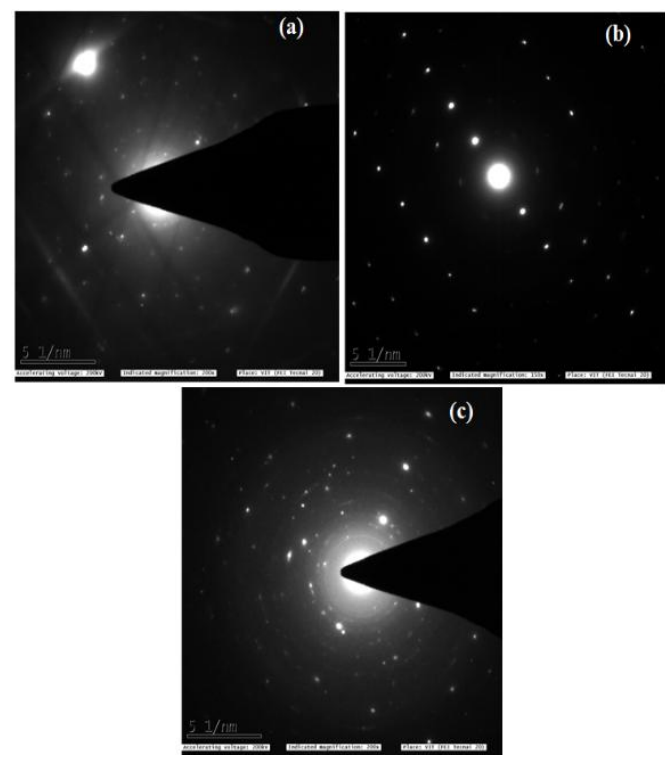

Fig. 4. SEAD Pattern of BCT-BST ceramics synthesized by (a) SSR (b) Sol-Gel Method and (c) Molten-Salt Method.

\subsection{Elastic and anelastic behaviour of BCT-BST}

The natural frequencies of BCT-BST ceramics are measured as a function of temperature (in the range $30^{\circ} \mathrm{C}$ to $120^{\circ} \mathrm{C}$ ), using composite piezoelectric oscillator technique. The effect of temperature on the natural frequency of the quartz transducer has been taken into account in evaluating the natural frequency of the ceramics at different temperatures. This data is used to obtain the longitudinal velocities, vl, of the ceramics.

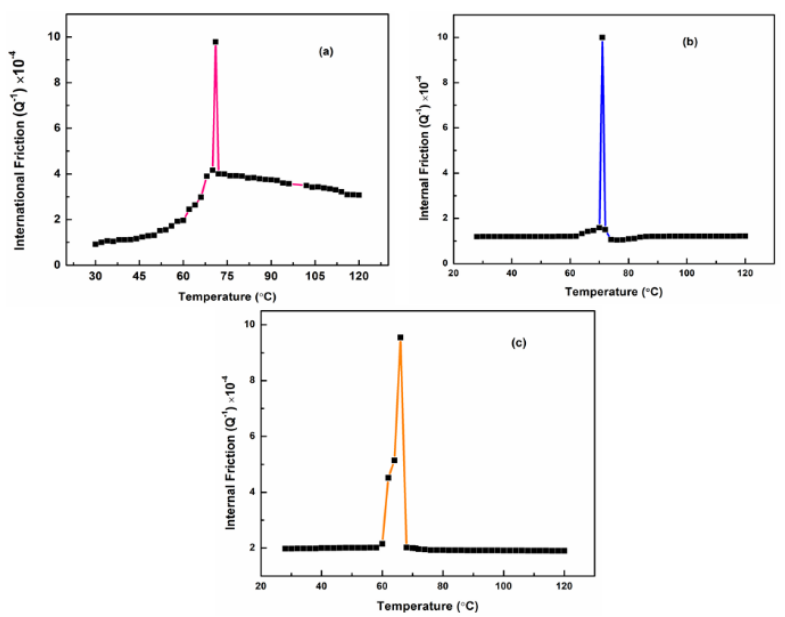

Fig. 5. Internal friction of BCT-BST ceramics synthesized by (a) SSR (b) Sol-Gel Method and (c) Molten-Salt Method.

Internal friction is a sensitive and effective measurement technique to detect the atomic arrangements especially defects, phase transition, domain wall motion and kinetics of atomic movements in the microstructure. Internal friction is the best tool to identify the phase transitions in ceramic materials. The prepared BCT-BST ceramic made into a bar of dimensions $22 \times 4 \times 4 \mathrm{~mm}$ is glued to $\mathrm{X}$-cut quartz transducer crystal of length of $22 \mathrm{~mm}$ and a cross section of 4 sq.mm. This forms the piezoelectric composite. The transducer natural frequency in the present study is 123.014 $\mathrm{kHz}$. and the mass of quartz

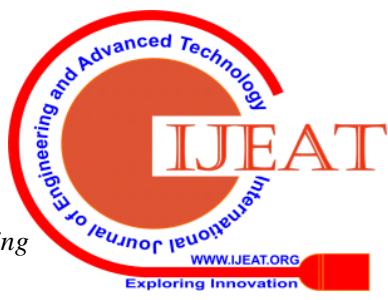


transducer is $1.0316 \mathrm{~g}$. The temperature dependence of internal friction Q-1 is shown in Fig. 5. All the three ceramics have exhibited an internal friction of about $10^{-3}$ at the transition temperature which is in good agreement with other Ba based ceramics [18]. With an increase in the temperature $\mathrm{Q}^{-1}$ peak is observed at the ferroelectric to paraelectric Curie transition point of $71^{\circ} \mathrm{C}$ for SSR and sol-gel synthesized BCT-BST ceramics. However the Curie transition for ceramic synthesized by molten-salt technique is noticed at $66^{\circ} \mathrm{C}$. Further the transition exhibits a broad peak with a kink which may be attributed to the relaxor ferroelectric nature of the ceramic or an improper ferroelastic transition mostly observed in barium based ceramics. According to Postnikov et al [19] fluctuating model of low frequency internal friction, at the phase transition interface it is assumed that thermally activated new nuclei will appear and grow by pinning the phase transition interface boundary. In addition it should be noticed that a maximum lattice strain is observed in the BCT-BST synthesized by molten salt technique (Table. 1). These attributes seems to explain the low temperature Curie transition in BCT-BST ceramics synthesized by molten salt technique. In other words, high lattice strain would be the result of pinning of phase transition boundary by the thermally activated nuclei. Fig. 6 shows the logitudinal modulus as a function of temperature of BCT-BST ceramics. The sharp dip of the longitudinal modulus in the temperature spectra again confirms the Curie phase transition point. The sharp peaks in internal friction and longitudinal modulus may be attributed to the movement of domain walls and rearrangement of point defects present in the prepared samples. Generally, the density of the domain walls increases when the materials are near the transition temperature. Temperature increases the mobility and density of domain walls and results in the high internal friction. According to Gridnov et al [20] if the density of domain wall is very high the acoustic wave interaction with the domain walls decreases the mobility of acoustic waves and the internal friction increases.
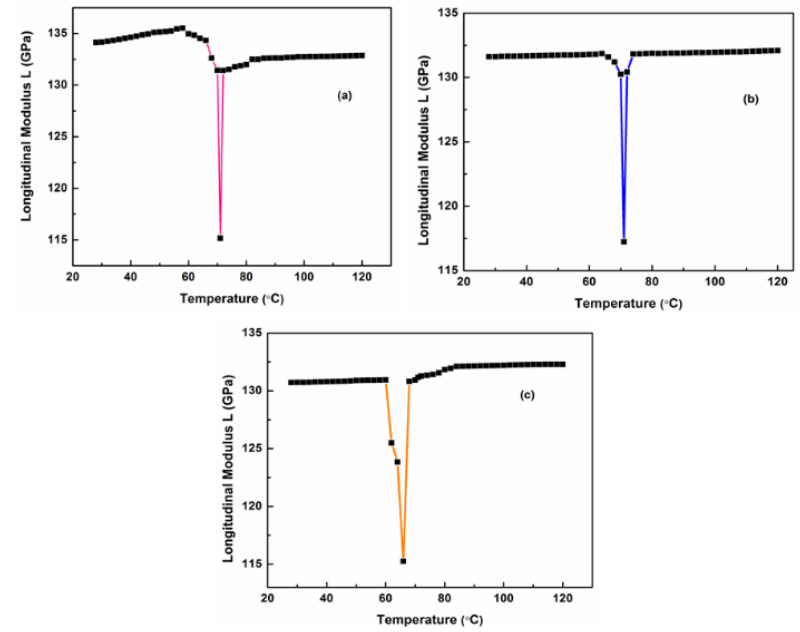

Fig. 6.Longitudinal modulus of BCT-BST ceramics synthesized by (a) SSR (b) Sol-Gel Method and (c) Molten-Salt Method

\section{CONCLUSION}

The BCT-BST ceramics are synthesized by solid state reaction method, sol-gel method and molten-salt method. The ceramics synthesized are all very dense and estimated porosity is $3 \%$. The cubic crystal structure is confirmed using the X-ray powder diffraction technique. The crystallite size as estimated from Scherrer equation revealed large crystals for SSR synthesized ceramic than sol gel and molten salt synthesized ceramics. The strain calculated using the W-H plot is found to increase for ceramics prepared by SSR, sol-gel method and molten-salt method respectively. TEM micrographs revealed the particle size in the range of 32 to $45 \mathrm{~nm}$. A large piezoelectric coefficient $\mathrm{d}_{33}$ of 619 to 623 is observed for the BCT-BST ceramics. The elastic and anelastic measurements resulted in an internal friction of $10^{-3}$ and a longitudinal modulus of approximately $115 \mathrm{GP}$ at the phase transition. The internal friction and longitudinal modulus behavior is explained on the basis of thermally activated pinning effects and lattice strains. With the knowledge of internal friction magnitude the ceramics potential as acoustic delay lines is envisioned.

Table- I: Physical properties of BCT-BST ceramics

\begin{tabular}{|c|c|c|c|c|c|c|c|c|c|}
\hline $\begin{array}{l}\text { Synthesis } \\
\text { methods }\end{array}$ & $\rho_{\mathrm{B}}\left(\mathrm{g} / \mathrm{cm}^{3}\right)$ & $\rho_{\mathrm{th}}\left(\mathrm{g} / \mathrm{cm}^{3}\right)$ & $\begin{array}{c}\mathrm{P} \\
(\%)\end{array}$ & $\begin{array}{c}\text { Crystallite } \\
\text { Size in nm } \\
\text { (XRPD) }\end{array}$ & $\begin{array}{c}\text { Scherrer } \\
\text { Strain }\left(\varepsilon^{*}\right)\end{array}$ & $\begin{array}{c}\text { W-H } \\
\text { Strain }\end{array}$ & $\begin{array}{l}\text { Particle } \\
\text { size in nm } \\
(\mathrm{TEM})\end{array}$ & $\begin{array}{c}\text { Piezoelectric } \\
\text { Coefficient } \\
\mathrm{d}_{33} \text { in } \mathrm{pC} / \mathrm{N} \\
\end{array}$ & $\begin{array}{c}\text { Piezoelectric } \\
\text { voltage }\left(\mathrm{G}_{33}\right) \\
\text { in } \mathrm{fC} / \mathrm{N}\end{array}$ \\
\hline SSR & 5.75 & 5.771 & 0.0379 & 50 & 0.1388 & 0.1327 & 60 & 623 & 69.22 \\
\hline Sol-gel & 5.72 & 5.742 & 0.0384 & 35 & 0.1889 & 0.1708 & 32 & 619 & 77.37 \\
\hline Molten-salt & 5.73 & 5.748 & 0.0313 & 35 & 0.211 & 0.2241 & 45 & 620 & 68.88 \\
\hline
\end{tabular}

5. Love, H. E. A. (1934), "Theory of Elasticity", Cambridge University Press, London and New York.

\section{REFERENCES}

1. Hans Jeffe (1958) "Piezoelectric ceramics" Journal of American Ceramic society, 41 (11), 299-506

2. Kailun Zoua, Yu Dan, Haojie Xu, Qing Feng Zhang, YinmeiLu, Haitao Huang, Yunbin He (2019) "Recent advances in lead-free dielectric materials for energy storage" Materials research bulletin 113, 190 - 201

3. Zhu, L. F., Zhang, B. P., Zhao, L., Li, S., Zhou, Y., Shi, X. C., Wang, N. (2016). 'Large Piezoelectric Effect of (Ba,Ca)TiO3 - X $\mathrm{Ba}(\mathrm{Sn}, \mathrm{Ti}) \mathrm{O} 3$ Lead-Free Ceramics'. Journal of the European Ceramic 36, 1017-24

4. Mason, P. W. (1985), "Physical Acoustics and the Properties of Solids", Van Nostrand, Princeton, New Jersey
6. Kolsky, H. (1953) "Stress Waves in Solids", Clarendon Press, Oxford.

7. Prager, W. (1961), "Introduction of Mechanics of Continua", Ginna nd journalsco., Boston, Massachussetts.

8. Sommerfeld, A. (1950), "Mechanics of Deformble Bodies", Academic Press, New York.

9. Bhimsenachar, J. (1962)," Internal Friction Behavior", Journal of Annamalai University, XXII, 14. 
10. Manson, P. W. (1965), (Ed.), "Physical Acoustics, Principles and Methods", Part B. Academic Press, New York, 3.

11. Manson, P. W. (1966), (Ed.), "Physical Acoustics, Principles and Methods", Part A. Academic Press, New York, 3.

12. Truel, R., Elbaum, C. and Chick, B. B. (1969), "Ultrasonic Methods in Solid State Physics", Academic Press, New York.

13. Seitz, F. and Turnbull, D. (1958), (Ed.) "Solid State Physics, Advances in Research and Applications", Academic Press, New York, 7.

14. Puškár, A. (2001), 'Internal Friction of Materials', Cambridge International Science Publishing.

15. Ramana, M. V., and Reddy, N. R. (2010), 'Internal friction and dielectric permittivity studies: barium lead titanate ferroelectric ceramics', Physica Scripta, 82(6), 065601.

16. Zhao, C., Hui, W., Jie, X. and Jiagang W. (2016), 'Composition-Driven Phase Boundary and Electrical Properties in $(\mathrm{Ba} 0.94 \mathrm{Ca} 0.06)(\mathrm{Ti1}-\mathrm{xMx}) \mathrm{O} 3$ (M = Sn, Hf, Zr) Lead-Free Ceramics' Dalton Transactions 45, 6466-80.

17. Yuvaraj, S., Nithya, V. D., Fathima, K. S., Sanjeeviraja, C., Selvan, G. K., Arumugam, S., Selvan, R. K. (2013), 'Investigations on the Temperature Dependent Electrical and Magnetic Properties of NiTiO3 by Molten Salt Synthesis'. Materials Research Bulletin 48(3), 1110-16.

18. S.A. Gridnev, I. I. Popov (2019), 'Effect of electronic subsystem on elastic and anelastic properties of ceramic $\mathrm{Ba}_{0.2} \mathrm{Sr}_{0.8} \mathrm{TiO}_{3}$ ', Ferroelectrics, 543, 130-136.

19. V. S. Postnikov, S. A. Gridnev, B. M. Darinskii and I. N. Sharshakov (1976) 'Internal friction at first order phase transition in solids', Il Nouvo Cimento B, 33B(1), 324-337.

20. Gridnev, S. A., (2007), 'Low-Frequency Shear Elasticity and Mechanical Losses in Ferroelastics', Ferroelectrics, 360,1-24.

\section{AUTHORS PROFILE}

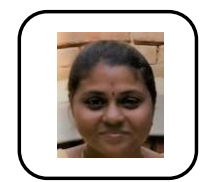

Dr. C. Pavithra has completed her doctorate in Physics in the year 2019. She has published 5 articles in peer reviewed international journals and 5 international conference series. Presently working as assistant professor at Department of Physics, Marudhar Kesari Jain College, Vaniyambadi, Vellore.

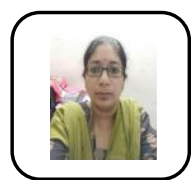

Dr. Madhuri W has got her Ph.D. degree from Sri Krishnadevaraya University, Anantapur in the year 2008. Since then she has published more than 60 articles in peer reviewed international journals and has supervised 4 Masters'thesis. students for their Ph.D and more than 10 students for their

Dr. Roopas Kiran S, is working as assistant professor in the department of Physics, VITAP, Andhra Pradesh. He did his Ph.D. from IIT Madras, and did Post Doc from Italy. He has published nearly 25 articles in peer reviewed international journals.

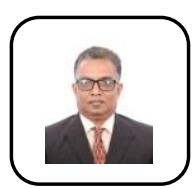

Dr. Arunai Nambiraj $\mathrm{N}$ is professor at centre for biometerials, cellular and molecular theranostics, VIT, Vellore. Prof. Arunai has supervised many Ph.D. , M. Phill and Master's students' thesis.

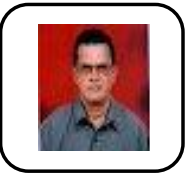

Prof. K. V. Siva Kumar has worked as professor at department of Physics, Sri Krishnadevaraya University, Anantapur. Prof. Siva Kuamr has published more than 150 articles in the field of ultrasonics and electroceramics. He also supervised more than 15 Ph.D., students and around 30 M.Phill students. 